\title{
Simple liver cysts and cystoid lesions in hepatic alveolar echinococcosis: a retrospective cohort study with Hounsfield analysis
}

\author{
Agata Engler ${ }^{1, a}$, Rong Shi ${ }^{1, a}$, Meinrad Beer ${ }^{1}$, Julian Schmidberger ${ }^{2}$, Wolfgang Kratzer ${ }^{2}$, Thomas F. E. Barth ${ }^{3}$, \\ Johannes Grimm ${ }^{3}$, Andreas Hillenbrand ${ }^{4}$, Doris Henne-Bruns ${ }^{4}$, Beate Gruener ${ }^{5}$, Ambros J. Beer ${ }^{6}$, \\ and Tilmann Graeter ${ }^{1, *}$ for the Echinococcosis Working Group, Ulm \\ ${ }^{1}$ Department of Diagnostic and Interventional Radiology, University Hospital Ulm, Albert-Einstein-Allee 23, 89081 Ulm, Germany \\ 2 Department of Internal Medicine I, University Hospital Ulm, Albert-Einstein-Allee 23, 89081 Ulm, Germany \\ 3 Department of Pathology, University Hospital Ulm, Albert-Einstein-Allee 23, 89081 Ulm, Germany \\ ${ }^{4}$ Department of General and Visceral Surgery, University Hospital Ulm, Albert-Einstein-Allee 23, 89081 Ulm, Germany \\ ${ }^{5}$ Department of Internal Medicine III, University Hospital Ulm, Albert-Einstein-Allee 23, 89081 Ulm, Germany \\ ${ }^{6}$ Department of Nuclear Medicine, Ulm University Hospital, Albert-Einstein-Allee 23, 89081 Ulm, Germany
}

Received 14 May 2019, Accepted 15 August 2019, Published online 30 August 2019

\begin{abstract}
Background. Alveolar echinococcosis (AE) is a rare zoonosis caused by the larval stage of the tapeworm Echinococcus multilocularis. AE lesions affect the liver in more than $98 \%$ of cases. AE lesions have various morphological characteristics that are described in the Echinococcus multilocularis Ulm classification for computed tomography (EMUC-CT). One of these characteristics is a cystoid portion. The aim of the study was to compare the density of simple hepatic cysts with cystoid portions of AE lesions classified on the basis of the EMUC-CT. Results. Hounsfield Unit (HU) measurements of the cystoid portions of all EMUC-CT type I-IV AE lesions $(n=155)$ gave a mean of $21.8 \pm 17.6$, which was significantly different from that of $2.9 \pm 4.5$ for the simple hepatic cysts $(p<0.0001)$. The difference between each of the individual AE types and simple hepatic cysts was also significant. In addition, the HU values of the cystoid portions in types I, II and IIIa/b and simple cysts were each significantly different from type IV $(p<0.0001)$. The HU measurements in type IV presented by far the highest mean. Conclusions. The significantly higher density measured in the cystoid portions of hepatic AE lesions offers a good means of differentiation from simple hepatic cysts.
\end{abstract}

Key words: Alveolar echinococcosis, Echinococcus multilocularis, Hepatic cysts, Density, Computed tomography, Classification.

Résumé - Kystes hépatiques simples et lésions cystö̈des dans l'échinococcose alvéolaire hépatique : une étude de cohorte rétrospective avec analyse de Hounsfield. Contexte. L'échinococcose alvéolaire (EA) est une zoonose rare causée par le stade larvaire du cestode Echinococcus multilocularis. Les lésions d'EA affectent le foie dans plus de $98 \%$ des cas. Les lésions d'EA ont différentes caractéristiques morphologiques décrites dans la classification d'Ulm pour la tomodensitométrie d'Echinococcus multilocularis (EMUC-CT). Une de ces caractéristiques est une portion cystoïde. Le but de l'étude était de comparer la densité de kystes hépatiques simples avec des portions cystoïdes de lésions d'AE classées sur la base du EMUC-CT. Résultats. La mesure en UH (Unités Hounsfield) des portions cystoïdes de toutes les lésions d'EA de type I-IV EMUC-CT $(n=155)$ a donné une moyenne de 21,8 $\pm 17,6$, significativement différente de celle de 2,9 \pm 4,5 pour les kystes hépatiques simples $(p<0,0001)$. La différence entre chacun des types d'EA individuels et les kystes hépatiques simples était également significative. De plus, les valeurs d'UH des portions de cystoïde des types I, II et IIIa/b et des kystes simples étaient chacune significativement différentes de celles du type IV $(p<0,0001)$. Les mesures d'UH pour le type IV présentaient de loin la moyenne la plus élevée. Conclusions. La densité significativement plus élevée mesurée dans les parties cystoïdes des lésions d'EA hépatiques offre un bon moyen de différenciation par rapport aux kystes hépatiques simples.

*Corresponding author: tilmann. graeter@uniklinik-ulm. de

${ }^{\mathrm{a}}$ Contributed equally.

This is an Open Access article distributed under the terms of the Creative Commons Attribution License (http://creativecommons.org/licenses/by/4.0), which permits unrestricted use, distribution, and reproduction in any medium, provided the original work is properly cited. 


\section{Abbreviations}

18F-FDG PET/CT 18-fluorodeoxyglucose positron-emission

$\mathrm{ABZ}$

$\mathrm{AE}$

BMZ

CT

DNA

EMUC-CT

EMUC-US

HCC

HU

MBZ

MPR

MRI

PACS

PAS

PNM

$r$

ROI

SD

TNM

US

WHO tomography/computed tomography Albendazole

Alveolar echinococcosis

Benzimidazole

Computed tomography

Deoxyribonucleic acid

Echinococcus multilocularis Ulm Classification for Computed Tomography

Echinococcus multilocularis Ulm Classification for Ultrasound

Hepatocellular carcinoma

Hounsfield unit

Mebendazole

Multiplanar reconstruction

Magnetic resonance imaging

Picture archiving and communication system

Parasite mass in the liver, neighbouring organs, metastasis

Correlation coefficient

Region of interest

Standard deviation

Primary tumour, lymph nodes, metastases

Ultrasound

World Health Organization
Periodic acid-Schiff

\section{Introduction}

Alveolar echinococcosis (AE) is a rare zoonosis caused by the larval stage of the fox tapeworm (Echinococcus multilocularis). Humans may become infected by ingesting contaminated food or direct contact with animals $[1,37]$. In such cases, humans act as aberrant intermediate hosts, as they do not belong to the natural life cycle of the parasite [10] and the formation of protoscolices is possible only in exceptional cases [8]. The liver is the organ most commonly affected [1], and the infiltrative growth of the lesions is similar to that of a malignant tumour $[18,33,34]$. The disease is the most dangerous parasitic zoonosis in Europe. Left untreated, it has a very high mortality rate $[1,10]$. One therapeutic option in terms of curative treatment is complete resection of the lesion, but this depends on the stage of the disease. Surgical excision may therefore contribute to cure [6]. If surgery is no longer an option, long-term pharmacotherapy with benzimidazole (BMZ) derivatives is indicated. Such treatment may inhibit further growth of the lesion and achieve a stable phase of the disease [4, 5, 10, 31]. Curative treatment of $\mathrm{AE}$ is only possible with early diagnosis $[6,9]$. WHO and the European Echinococcosis Registry developed a classification similar to the TNM $(\mathrm{T}=$ Primary tumour, $\mathrm{N}=$ lymph nodes, $\mathrm{M}=$ metastases) system for malignant tumours to allow for better assessment of the disease stage. Diagnostic imaging forms the basis of the so-called PNM $(\mathrm{P}=$ parasitic mass in the liver, $\mathrm{N}=$ involvement of neighbouring organs, $\mathrm{M}=$ metastasis) classification. The classification allows for standardised staging upon initial diagnosis, which ensures optimal treatment [20]. Besides the case history, the

diagnosis of $\mathrm{AE}$ requires a combination of imaging techniques such as ultrasound (US), computed tomography (CT), magnetic resonance imaging (MRI), and $18 \mathrm{~F}$ fluorodeoxyglucose positron emission tomography/computed tomography (18F-FDG PET/CT), and the results of immunodiagnostics (specific serology). Histopathological findings and molecular DNA identification are used to confirm the diagnosis [39].

The MRI classification developed by Kodama et al. has been available since 2003 [21] and describes five different types of $\mathrm{AE}$ lesions, including two patterns of the cystic component: small round cysts and large and/or irregular cysts. The Echinococcus multilocularis Ulm Classification for Ultrasound (EMUC-US) was introduced by Kratzer et al. in 2015. This classification also enables the differentiation of five different morphological types of hepatic AE lesions [23], including pseudocystic and metastatic-like forms. The Echinococcus multilocularis Ulm classification for computed tomography (EMUC-CT) introduced by Graeter et al. in 2016 [12] offers the basis for the systematic description of the CT morphology of $\mathrm{AE}$ lesions of the liver. Five primary morphological types can be distinguished. Type I: diffusely infiltrating; II: primarily circumscribed tumour-like; III: primarily cystoid (IIIa - intermediate or IIIb - widespread, depending on extent); IV: small cystoid/metastatic; V: mainly calcified. Subcriteria with respect to the presence of cystoid portions apply to types I and II, while subcriteria for the primarily cystoid type III concern the presence of a more solid margin. In addition, 1 of 6 separately defined calcification patterns is allocated to each primary morphological type, with the exception of type $\mathrm{V}$, to allow for comprehensive description of the lesion. These patterns are: no calcifications, feathery calcifications, focal calcifications, a central calcification, diffuse calcifications and calcifications primarily at the edge. The pattern "with a central calcification" is a feature of type IV only (Fig. 1).

Alveolar echinococcosis (AE) has to be distinguished from cystic echinococcosis (CE), caused by the larvae of the dog tapeworm (Echinococcus granulosus). $\mathrm{CE}$ is the most common zoonosis [8] but follows a significantly more benign course than AE. CE involves mainly the liver and lungs [27].

Hepatic cysts are the most common incidental finding among focal liver lesions seen on contrast-enhanced CT [36]. Simple cysts are congenital in origin, developing from abnormal biliary tract cells and contain clear, sometimes yellowish to bilecoloured fluid [35]. They appear on CT scans as sharply demarcated hypodense lesions with a density of 0-30 Hounsfield Units (HU), i.e. isodense with fluid $[29,30]$. After the administration of contrast medium, they show no enhancement and no relevant change in density $(>10 \mathrm{HU})$. If there has been bleeding into the cyst or the cyst contains protein, the density may already be more than $30 \mathrm{HU}$ in the non-contrast scan [29]. Cysts are therefore important in the differential diagnosis of $\mathrm{AE}$ lesions. Any cystic hepatic lesion has to be assessed for further benign and malignant changes. Possible lesions include nonneoplastic lesions such as liver abscesses, CE cysts, Caroli disease, bilomas, as well as neoplastic lesions such as cystadenomas, cystadenocarcinomas, or necrosis in hepatocellular carcinomas (HCC) or metastases [21, 24, 30-32].

The present study compares the density of simple hepatic cysts with the density of cystoid portions of various EMUC-CT 


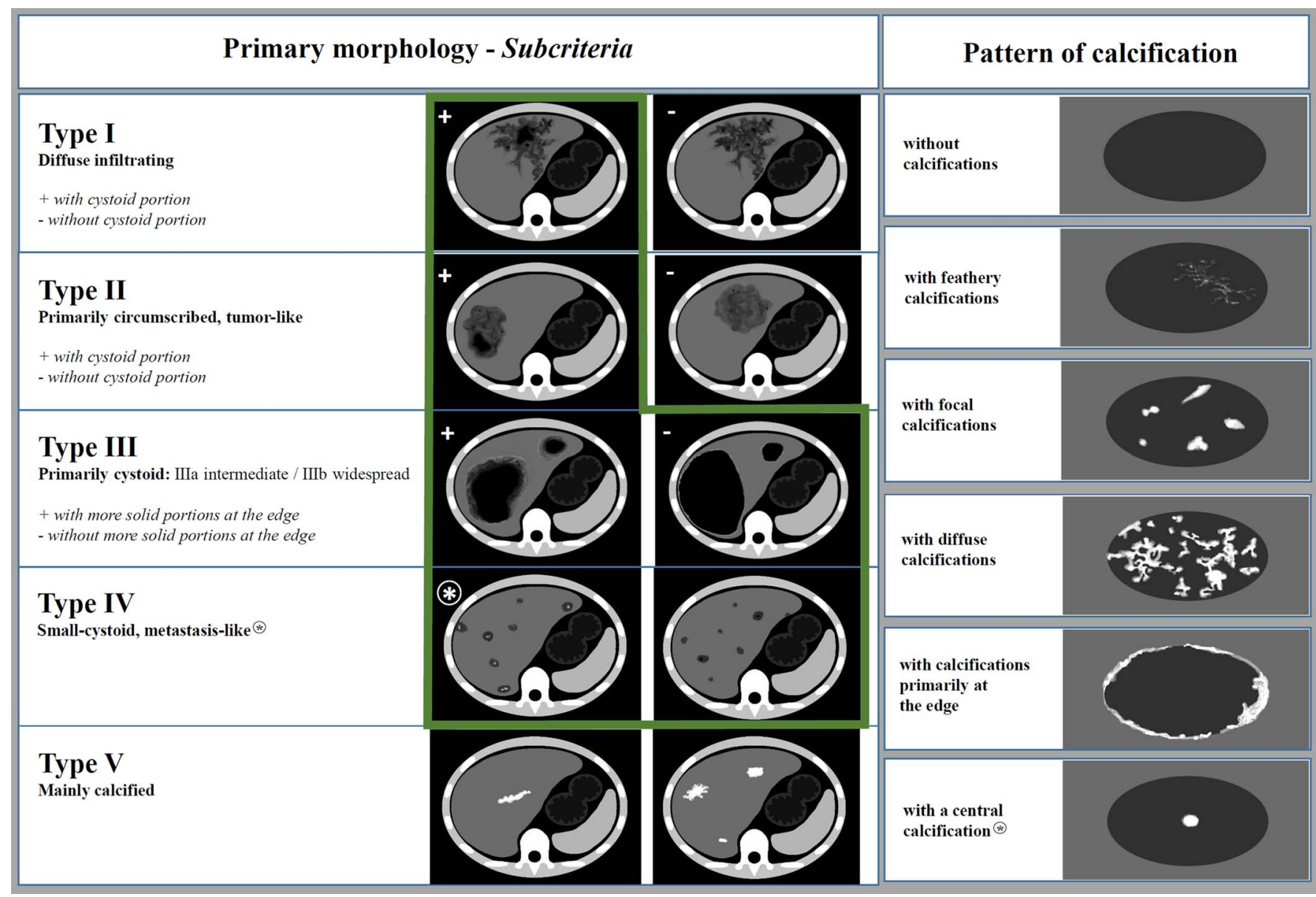

Figure 1. Overview of the EMUC-CT after Graeter et al. Left: Primary morphological types I-V and their subcriteria (applicable to types I, II and III). Right: Patterns of calcification. (EMUC-CT = Echinococcus multilocularis Ulm Classification for Computed Tomography). The two columns of the classification are primarily considered separately and can then in principal be freely combined. There are two exceptions: The pattern of calcification "with a central calcification*" can only occur with primary morphology type IV* and primary morphology type V is not further characterised by a pattern of calcification. Lesions of types I and II with cystoid portions as well as types III and IV were evaluated (marked with a green frame).

types on the basis of HU measurements. This should further characterise the various primary morphological types of $\mathrm{AE}$, and in some cases could help to make a differential diagnostic distinction especially between particular AE lesions with a primarily cystoid aspect, such as type III without more solid portions at the edge as well as type IV, and simple hepatic cysts.

\section{Materials and methods}

\section{Study design}

Ulm University hospital administers a national database for alveolar echinococcosis. From this database, we retrospectively extracted data for patients who had an 18F-FDG PET/CT scan in the Department of Nuclear Medicine between May 2005 and December 2017. All cases had confirmed or probable AE according to the WHO case definition but had not undergone surgery on the liver by the time of the initial PET/CT examination. We classified the hepatic lesions using the EMUC-CT. Cases that were classified as type I or type II without cystoid portions or as the predominantly calcified type $\mathrm{V}$ were excluded from the study.

The study population included patients who were treatmentnaïve at the time of the examination, as well as those who had already started treatment with BMZ derivatives: albendazole (ABZ) or mebendazole (MBZ). This resulted in a study population of $n=155$ subjects.

The control group consisted of patients without AE or CE, who had an 18F-FDG PET/CT scan performed between October 2014 and December 2017 for other reasons (e.g. cancer screening, staging of a known primary tumour, looking for a focus of infection), in whom hepatic cysts had been detected. The control group with simple cysts consisted of $n=78$ patients. The whole study population therefore consisted of $n=233$ patients.

Patient data collected from both groups included sex and age. Data were extracted retrospectively from the digital medical records of the database system (Systems, Applications and Products in Data Processing [SAP]) of the Ulm University Hospital according to the medical letter drafted at the time of the examination. 
Examinations were carried out with two different PET/CT scanners: Discovery LS from General Electric (CT: Lightspeed plus; collimation $4 \times 5.0 \mathrm{~mm}$ ) and Biograph $\mathrm{mCT}-\mathrm{S}(40)$ from Siemens (CT: Somatom Definition AS 40; collimation $16 \times 1.2 \mathrm{~mm})$. The CT data from each PET/CT examination were used to evaluate density. The portal venous phase $(n=135 ; 87.1 \%)$ and the non-contrast dataset $(n=20 ; 12.9 \%)$ were both evaluated for the AE patients; only the contrastenhanced scans were used for the control group. Images obtained elsewhere were not taken into consideration.

Measurements in the patients with $\mathrm{AE}$ were performed twice, by two specialty trainee radiologists with different experience (about 3 years and 6 years, respectively). They performed independent assessments from the multiplanar reconstructions (MPRs) with $4 \mathrm{~mm}$ or $5 \mathrm{~mm}$ slices using the Picture Archiving and Communication System (PACS) programme. Measurements in the control group were carried out once only (by the specialty trainee with about 6 years' experience). For both groups, the largest cyst or the largest hepatic AE lesion with a cystoid portion was selected as the reference lesion. Its location in the lobe and segment of the liver, its largest diameter and the density of the cyst or cystoid portion of the lesion were recorded. For this purpose, the examiner selected a transverse slice with the lowest optical density in the area of the selected lesion and made two separate measurements in manually outlined round or elliptical regions of interest (ROIs) with an area of $30 \pm 1 \mathrm{~mm}^{2}$ (Fig. 2). The density was measured in $\mathrm{HU}$, recording the minimum and maximum values and calculating the mean and standard deviation $(S D)$. Data were collected in tabular form using Microsoft Excel.

\section{Ethics statement}

The study was carried out in accordance with the Declaration of Helsinki. The study protocol was approved by an independent local ethics committee (Application no 409/15). As the study was retrospective, it was not possible to obtain written informed consent from the patients. For this reason, all data were anonymised prior to the statistical analysis.

\section{Statistical analysis}

We used SAS Version 9.4 software (SAS Institute Inc., Cary, NC, USA) for the statistical analysis of the data. The Shapiro-Wilk test was used to check the normal distribution of the data. Differences between two independent groups were tested with the Wilcoxon-Mann-Whitney test. A $p$-value of $5 \%(<0.05)$ was considered statistically significant and was reported to four decimal places. Correlation between selected values was assessed using the Spearman's rank correlation coefficient $\left(r_{\mathrm{Sp}}\right)$.

\section{Results}

\section{Sex and age in the study and control groups}

The study group of patients with AE comprised 90 women $(58.0 \%)$ and 65 men (42.0\%) aged between 18 and 87 years, with a mean age of $54.5 \pm 17.4$ years at the time of the

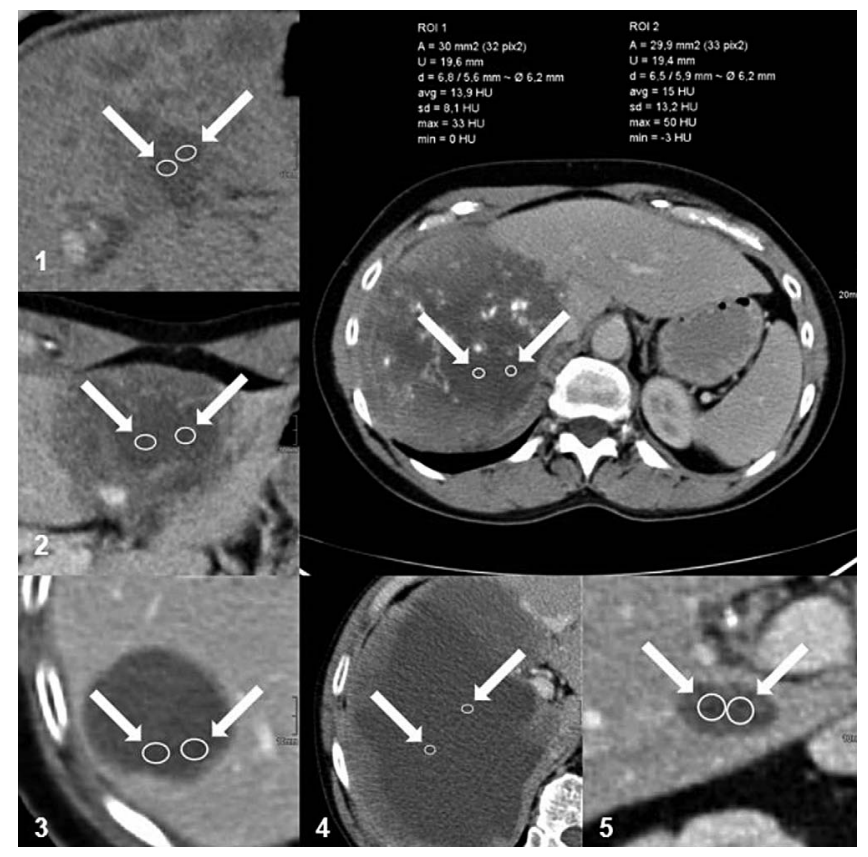

Figure 2. Example of density measurement in the cystoid portions of AE lesions on CT. Left and below: in each case, two enlarged ROIs within the cystoid portions of AE lesions of different primary morphological types: (1) Type I "diffuse infiltrating with cystoid portion"; (2) Type II "primarily circumscribed tumour-like with cystoid portion"; (3) Type IIIa "primarily cystoid intermediate without solid portion at the edge"; (4) Type IIIb "primarily cystoid widespread with solid portion at the edge"; (5) Type IV "small cystoid/metastatic". Above right: two ROIs with surface data and HU values (max, min, mean and standard deviation) within the cystoid portion of an $\mathrm{AE}$ lesion involving both lobes of the liver. $(\mathrm{AE}=$ alveolar echinococcosis; $\mathrm{CT}=$ computed tomography; $\mathrm{HU}=$ Hounsfield unit; ROI $=$ region of interest).

examination. The control group of patients with hepatic cysts consisted of 46 women $(59.0 \%)$ and 32 men (41.0\%) aged between 38 and 76 years. The mean age was $62.2 \pm 8.7$ years.

\section{Lesion size in the study and control groups}

Lesions in patients with AE were between 8 and $210 \mathrm{~mm}$ in size, with a mean of $79.3 \pm 48.1 \mathrm{~mm}$. The cysts were significantly smaller with a diameter between 13 and $100 \mathrm{~mm}$ and a mean of $30.9 \pm 16.6 \mathrm{~mm}(p<0.0001)$ (Table 1$)$.

\section{Distribution of the lesions in the lobes of the liver in the study and control groups}

In the study group, the largest $\mathrm{AE}$ lesions were found in the right lobe of the liver in 86 cases $(55.4 \%)$ and in the left lobe in 36 patients $(23.2 \%)$. In 33 cases $(21.2 \%)$, the $\mathrm{AE}$ lesions traversed the border between the lobes and were therefore present in both lobes. Figures for the simple cysts were 34 $(43.5 \%)$ in the right lobe and $44(56.4 \%)$ in the left lobe. None of the cysts extended into both lobes. The difference in distribution of the lesions between both groups was statistically significant $(p<0.0001)$. 
Table 1. Patient characteristics in the study group $(n=155)$ and the control group $(n=78)$.

\begin{tabular}{|c|c|c|}
\hline \multirow[t]{2}{*}{ Characteristic } & $\begin{array}{l}\text { Study group } \\
(n=155)\end{array}$ & $\begin{array}{l}\text { Control group } \\
\quad(n=78)\end{array}$ \\
\hline & \multicolumn{2}{|c|}{$N(\%)$} \\
\hline \multicolumn{3}{|l|}{ Sex } \\
\hline Male & $65(42.0)$ & $32(41.0)$ \\
\hline Female & $90(58.0)$ & $46(59.0)$ \\
\hline \multicolumn{3}{|l|}{ Age at time of examination } \\
\hline$<18$ years & $0(0.0)$ & $0(0.0)$ \\
\hline $18-50$ years & $61(39.3)$ & $8(10.2)$ \\
\hline $51-60$ years & $30(19.3)$ & $26(33.3)$ \\
\hline $61-70$ years & $27(17.4)$ & $28(35.9)$ \\
\hline$>70$ years & $37(23.8)$ & $16(20.5)$ \\
\hline \multicolumn{3}{|l|}{ Pharmacotherapy } \\
\hline No treatment & 49 (31.6) & \\
\hline$\leq 35$ days & $21(13.5)$ & \\
\hline$>35$ days & $65(41.9)$ & \\
\hline \multirow[t]{2}{*}{ Treatment and interruption } & $20(12.9)$ & \\
\hline & \multicolumn{2}{|c|}{$\begin{array}{l}\text { Mean } \pm S D \\
\text { Min - Max }\end{array}$} \\
\hline HU value (unit) & $\begin{array}{c}21.8 \pm 17.6 \\
-16.4-76.9\end{array}$ & $\begin{array}{c}2.9 \pm 4.5 \\
-9.9-10.8\end{array}$ \\
\hline Age at examination (years) & $\begin{array}{c}54.5 \pm 17.4 \\
18-87\end{array}$ & $\begin{array}{l}62.2 \pm 8.7 \\
38-76\end{array}$ \\
\hline $\begin{array}{l}\text { Size of the space-occupying } \\
\text { lesion }(\mathrm{mm})\end{array}$ & $\begin{array}{l}79.3 \pm 48.1 \\
8-210\end{array}$ & $\begin{array}{c}30.9 \pm 16.6 \\
13-100\end{array}$ \\
\hline
\end{tabular}

$\mathrm{HU}=$ Hounsfield unit; $S D=$ standard deviation; $\max =$ maximum; $\min =$ minimum.

\section{Primary morphology of the AE lesions}

The frequency of the primary morphology types in the 155 AE lesions was type I $25.8 \%(n=40)$, type II $14.8 \%(n=23)$, type III 31.6\% $(n=49)$ (of which $16.7 \%$ [ $n=26]$ were type IIIa and $14.8 \%[n=23]$ were type IIIb) and type IV $27.7 \%(n=43)$. The type III lesions were subdivided: $63.2 \%(n=31)$ with a solid portion at the edge and $36.7 \%(n=18)$ without a solid portion at the edge. Type IIIa consisted of $46.2 \%(n=12)$ with solid portion at the edge and $53.8 \%(n=14)$ without a solid portion at the edge, while type IIIb consisted of $82.6 \%$ $(n=19)$ with a solid portion at the edge and $17.4 \%(n=4)$ without a solid portion at the edge.

\section{Calcification pattern of the AE lesions}

The calcification pattern of the lesions was as follows: no calcifications $n=40(25.8 \%)$, feathery calcifications $n=19(12.2 \%)$, diffuse calcifications $n=36$ (23.2\%), focal calcifications $n=21(13.5 \%)$, calcifications primarily at the edge $n=23(14.8 \%)$, and a central calcification $n=16$ $(10.3 \%)$.

\section{HU values in the study and control groups}

The inter-rater reliability for the measurement of the $\mathrm{AE}$ lesions was $92 \%$.

Density measurements of the cystoid portions of each primary morphological type gave the following means: type I
Table 2. HU values in the study group $(n=155)$ according to primary morphological type and in the control group $(n=78)$.

\begin{tabular}{lccrl}
\hline Morphology & $N$ & $\begin{array}{c}\text { Mean HU } \\
\text { value } \pm S D\end{array}$ & $\begin{array}{c}\text { Min - Max } \\
\text { HU value }\end{array}$ & $p$-value \\
\hline Type I & $n=40$ & $14.9 \pm 11.5$ & $-16.4-52.5$ & $p<0.0001$ \\
Type II & $n=23$ & $17.7 \pm 14.2$ & $-3.0-63.1$ & \\
Type III & $n=49$ & $13.5 \pm 11.5$ & $-3.4-49.5$ & \\
Type IIIa & $n=26$ & $16.5 \pm 13.8$ & $-3.4-49.5$ & \\
Type IIIb & $n=23$ & $10.3 \pm 7.0$ & $-3.3-24.3$ & \\
Type IV & $n=43$ & $39.8 \pm 17.2$ & $3.0-76.9$ & \\
Cysts & $n=78$ & $2.9 \pm 4.5$ & $-9.9-10.8$ & \\
\hline
\end{tabular}

$\mathrm{HU}=$ Hounsfield unit $S D=$ standard deviation; $\max =$ maximum; $\min =$ minimum. $p<0.05$ statistically significant.

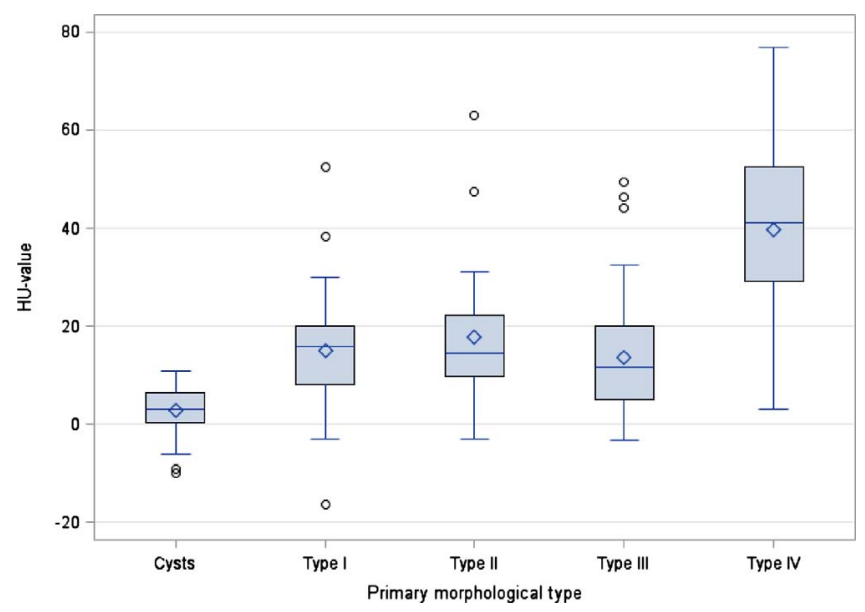

Figure 3. Box plot showing the HU values and spread of the EMUC-CT primary morphological types and cysts.

$14.9 \pm 11.5 \mathrm{HU}$, type II $17.7 \pm 14.2 \mathrm{HU}$, type III $13.5 \pm 11.5 \mathrm{HU}$ (type IIIa $16.5 \pm 13.8 \mathrm{HU}$ and type IIIb $10.3 \pm 7.0 \mathrm{HU}$ ). Type IV showed by far the highest density with $39.8 \pm 17.2 \mathrm{HU}$; this was significantly different from the other types (I, II and III) $(p<0.0001)$ (Table 2). Density measurements of all the cystoid portions of the AE lesions (types I-IV) together gave a mean of $21.8 \pm 17.6 \mathrm{HU}$ with a spread between -16.4 and $76.9 \mathrm{HU}$. This was significantly different $(p<0.0001)$ from the simple hepatic cysts, which showed a much lower density at a mean of $2.9 \pm 4.5 \mathrm{HU}$ (min $-\max -9.9-10.8 \mathrm{HU}$ ). The cystoid portions of the individual AE types (I-IV) also differed significantly in density from the simple cysts $(p<0.0001)$ in each case (Table 3$)$. In addition, there were significant differences in the $\mathrm{HU}$ values of the cystoid portions in types I, II and IIIa/b and the simple cysts compared with type IV $(p<0.0001)$ (Fig. 3, Table 3).

\section{Correlations within the study group}

Density measurements of the cystoid portions of AE lesions showed a correlation between the pattern of the primary morphology and the HU value $(r=0.43139)$. Comparison of the size of the lesion with the density demonstrated a high correlation between the two parameters $(r=-0.60837)$. The smaller 
Table 3. Difference in the HU values of primary morphological types I-IV compared with cysts, as well as of primary morphological types IIII and cysts compared with type IV.

\begin{tabular}{|c|c|c|c|c|c|c|}
\hline \multirow[t]{2}{*}{ Morphology } & \multicolumn{6}{|c|}{$\begin{array}{l}\text { Mean HU value } \pm S D \\
\text { Min }- \text { Max HU value }\end{array}$} \\
\hline & Type X & Cysts & $p$-value & Type X & Type IV & $p$-value \\
\hline Type I & $\begin{aligned} & 14.9 \pm 11.5 \\
- & 16.4-52.5\end{aligned}$ & $\begin{array}{r}2.9 \pm 4.5 \\
-9.9-10.8\end{array}$ & $<0.0001$ & $\begin{aligned} & 14.9 \pm 11.5 \\
- & 16.4-52.5\end{aligned}$ & $\begin{array}{c}39.8 \pm 17.2 \\
3.0-76.9\end{array}$ & $<0.0001$ \\
\hline Type II & $\begin{array}{l}17.7 \pm 14.2 \\
-3.0-63.1\end{array}$ & $\begin{array}{r}2.9 \pm 4.5 \\
-9.9-10.8\end{array}$ & $<0.0001$ & $\begin{array}{l}17.7 \pm 14.2 \\
-3.0-63.1\end{array}$ & $\begin{array}{c}39.8 \pm 17.2 \\
3.0-76.9\end{array}$ & $<0.0001$ \\
\hline Type III & $\begin{array}{l}13.5 \pm 11.5 \\
-3.4-49.5\end{array}$ & $\begin{array}{r}2.9 \pm 4.5 \\
-9.9-10.8\end{array}$ & $<0.0001$ & $\begin{array}{l}13.5 \pm 11.5 \\
-3.4-49.5\end{array}$ & $\begin{array}{l}39.8 \pm 17.2 \\
3.0-76.9\end{array}$ & $<0.0001$ \\
\hline Type IV/cysts & $\begin{array}{l}39.8 \pm 17.2 \\
3.0-76.9\end{array}$ & $\begin{array}{r}2.9 \pm 4.5 \\
-9.9-10.8\end{array}$ & $<0.0001$ & $\begin{array}{r}2.9 \pm 4.5 \\
-9.9-10.8\end{array}$ & $\begin{array}{l}39.8 \pm 17.2 \\
3.0-76.9\end{array}$ & $<0.0001$ \\
\hline
\end{tabular}

$\mathrm{HU}=$ Hounsfield Unit; $S D=$ standard deviation; $\max =\operatorname{maximum}$; $\min =$ minimum. $p<0.05$ statistically significant.

the lesion, the higher the $\mathrm{HU}$ value. Both of these comparisons were statistically significant $(p<0.0001)$. In contrast, comparison of the underlying calcification patterns and the density of the cystoid portions gave a low correlation coefficient $(r=-0.04004)$ and was not significant $(p<0.6220)$.

\section{Discussion}

Imaging plays a key role in the diagnostic investigation of $\mathrm{AE}[1,5,28]$. As earlier studies have shown, AE lesions show a broad spectrum of morphological characteristics on ultrasound, CT and MRI allowing various types to be distinguished. One criterion is the possible presence of liquid components of the lesions. The EMUC-US [23] defined a pseudocystic pattern, and the MRI classification from Kodama et al. [21] describes small round cysts as well as large and/or irregular cysts. Nevertheless, the classical picture of a cyst in the strictest sense is not seen in AE but is possibly reflected as "small cysts" in the sense of alveoli. The EMUC-CT has established the term "cystoid" for the confluent hypodense areas of the lesions.

When cyst-like lesions or parts of lesions occur in AE, their appearance in the different imaging modalities may present a real challenge in the differential diagnostic distinction from simple cysts, the most common focal liver lesions [17, 24, 36]. Distinguishing these two entities from hepatic Echinococcus granulosus infestations is a further challenge [27, 38]. It must be remembered that the different types of CE lesion may sometimes closely resemble simple cysts, depending on the stage of the disease [28]. The serological evidence that contributes to the diagnosis may be conflicting in both parasitic infestations. It may therefore delay an early diagnosis or even foster a misdiagnosis. Positive serology alone is not sufficient to confirm the diagnosis of echinococcosis [39].

The CT modality offers the possibility of determining the density of structures quantitatively by measuring $\mathrm{HU}$ values [15]. Already in 1985, Didier et al. reported CT findings in a small population of 24 patients with hepatic AE lesions showing on the one hand heterogeneous hypodense areas without contrast medium enhancement with densities of 20-40 HU in $92 \%$ of cases, and on the other hand pseudocystic areas with densities of $0-10 \mathrm{HU}$ within the hypodense regions with a "geographical map" pattern in $40 \%$ of cases [9]. Liu et al. found that some patients with $\mathrm{AE}$ had cystic cavities with densities between 3 and $10 \mathrm{HU}$ on CT after ABZ therapy, concluding that these cavities contained serous fluid [26]. Reuter et al. too reported CT findings of central hypodense necrotic areas and densities of $0-25 \mathrm{HU}$ in AE lesions [31]. The present study is the first to compare simple hepatic cysts with the cystoid portions of various EMUC-CT types of AE lesion on the basis of the $\mathrm{HU}$ value in a large patient population $(n=155)$.

The ratio of female to male subjects in the study group with $\mathrm{AE}$ (58.0\% women and $42.0 \%$ men) reflects previous observations that women are more commonly affected [13, 14, 16, 19, $20,22,31,37]$. Nevertheless, it has to be remembered that our exclusion criteria (AE primary morphology type I and type II without cystoid portions, type $\mathrm{V}$, patients who had undergone surgery and patients whose diagnostic imaging had been performed elsewhere) meant that not all the patients in the local Echinococcus database were included in the study. The presence of more female patients in the control group agrees with the prevalence of hepatic cysts found in other studies to date, although these were not always statistically significant $[11,17$, 25, 35].

With respect to the density of the cystoid portions of the hepatic AE lesions overall compared with the density of simple hepatic cysts, our study showed that the cystoid components in AE have significantly higher HU values than hepatic cysts $(21.8 \pm 17.6 \mathrm{HU}$ vs $2.9 \pm 4.5 \mathrm{HU})$. This difference was also significant when comparing the simple hepatic cysts with each of the individual AE types. This result is clinically relevant, as a distinction between the two entities may be crucial in the context of differential diagnosis, especially with regard to the primarily cystoid AE types III and IV. Furthermore, if cysts occur polycyclically or partially septated, they can assume very bizarre formations in $\mathrm{CT}$, so that the differentiation from the other manifestations of AE must also be made. The HU value measurement can be one mosaic stone in the first approach to differential diagnosis in this context. The present results also emphasise the significance of the terminology selected for the EMUC-CT. Types I and II of the five primary morphological lesions may contain hypodense regions as a subcriterion, while such areas are already included in the primary description of 


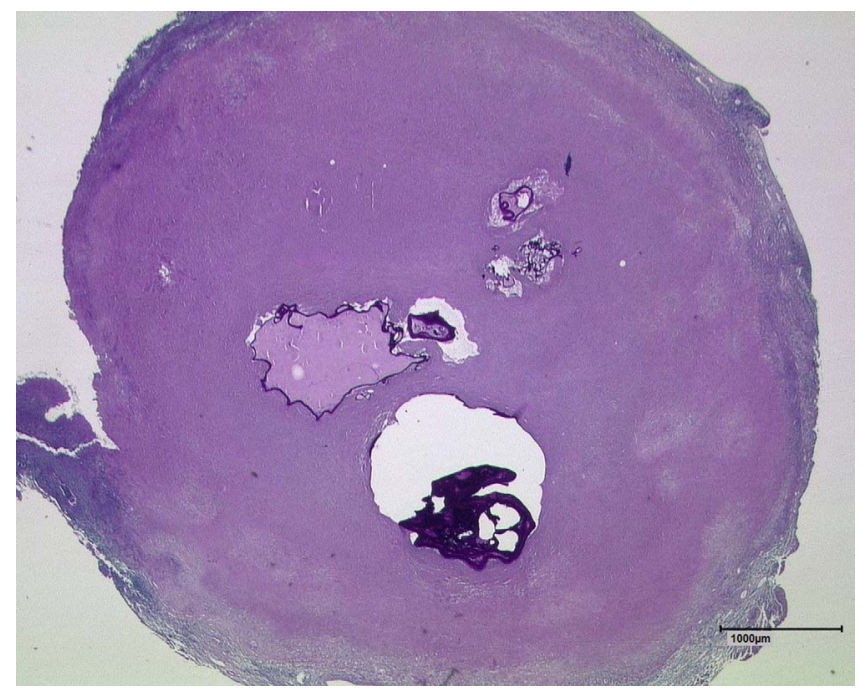

Figure 4. Section of a type IV AE lesion with central alveoli after PAS staining at $12.5 \times$ magnification. Note the wide surrounding area of solid necrosis. $(\mathrm{AE}=$ alveolar echinococcosis, $\mathrm{PAS}=$ periodic acid-Schiff)

types III and IV, being correctly designated "cystoid" rather than "cystic".

The cystoid portions in diffuse infiltrating type I had a mean density of $14.9 \pm 11.5 \mathrm{HU}$ and in primarily circumscribed tumour-like type II a mean density of $17.7 \pm 14.2 \mathrm{HU}$. In relation to the whole study population, these types had a moderately high increase in density. It is possible that the cystoid portions in these two groups either still consisted of alveolar conglomerations with intervening septa or of areas of still denser necrosis with incipient liquefaction. Septa included in the measurements or areas of necrosis undergoing initial liquefaction but still relatively protein rich could lead to an increased density of this nature. The mean $\mathrm{HU}$ value for type III (type IIIa/b together) was $13.5 \pm 11.5 \mathrm{HU}$, with values tending to be lower than those of types I and II. Type IIIb, which is primarily cystoid with extensive lesions, notably had the lowest density of any AE type at $10.3 \pm 7.0 \mathrm{HU}$ and was therefore closest to the hepatic cysts. This can be explained by the fact that the cystoid portions here comprised a single area several centimetres across, consistent with necrosis in an advanced stage of liquefaction, as was discussed when the classification was established in 2016 [12]. Evidence that type III is actually older AE lesions has been supplied by a recent international comparative study [13]. The relationship that we observed between the size of the lesion and the density of the cystoid portions shows a high correlation $(r=-0.60837)$ and we can conclude: the smaller the lesion, the higher the HU value. This would also support the hypothesis outlined above.

Regarding the correlation between lesion size and density, the very small type IV consequently has an exceptional position in the five EMUC-CT primary morphology types. This type of lesion showed by far the highest density, with a mean of $39.8 \pm 17.2 \mathrm{HU}$. Type IV was therefore significantly different not only from simple cysts but also from the cystoid portions of types I, II and III. Type IV lesions usually consist of one small alveolus (sometimes even a few) surrounded by solid necrosis of varying width (Fig. 4). In the CT images, this wide band of necrosis also appears hypodense, although to a lesser extent than the alveoli. Given a sufficiently pronounced central alveolus, these lesions may therefore also furnish sensitive evidence in T2-weighted MRI [2]. The existing dichotomy in the structure of type IV lesions leads to higher density values than found in the other types: density measurements within the ROI give lower HU values if an alveolus of a certain size is present, whereas they increase if the alveolus is very small or even absent. The two main lesion characteristics of type IV are described as small cystoid/metastatic according to the previous EMUC-CT terminology. As the term is intended to describe only the morphological appearance and not any metastatic potential of these lesions, we propose that in future it should be "small cystoid/metastasis-like", which is less likely to be misunderstood.

Finally, the aspect of the different densities within the cystoid portions of the various types that we have discussed above, fits well with the evolutionary hypothesis of AE lesions outlined in a recent multicentre study [13]. In this context, there are intermodal parallels to important observations made with Kodama's MRI classification [3, 21]. It would be interesting for future studies to compare CT and MRI findings, as current knowledge suggests parallels between the classification of the cystoid portions in EMUC-CT types I, II and III and types 1, 3 and 5 of Kodama's classification. Such a study could also demonstrate that the Kodama classification does not yet have an equivalent to the small initial, potentially either intensifying or involuting, type IV of the EMUC-CT.

One limitation of the study was the significant age difference between the study group and the control group. This difference may be explained firstly by the fact that the prevalence of hepatic cysts increases with age, and cysts in patients below the age of 40 are a rarity $[7,11,17,25,35]$. Secondly, the PET/ CT scans were frequently carried out in the control group because of cancer; this patient group is also one of more advanced age. Furthermore the study does not provide a juxtaposition of CT morphology and histopathology.

\section{Conclusions}

Depending on the morphology, cystoid components may be found in hepatic AE lesions. For this reason, particular $\mathrm{AE}$ lesions belong, besides initial stages of $\mathrm{CE}$, to the most important conditions in the differential diagnosis of the most common focal liver lesions, benign hepatic cysts. With significantly higher values, density measurements of cystoid hepatic $\mathrm{AE}$ lesions offer a good opportunity for distinguishing them from simple hepatic cysts. There is a relationship between lesion size and density value, possibly as the expression of lesions in different stages of advancement. The highest density of type IV, which is significantly different from all the other types of lesions, fits the structure of this lesion which, in addition to hypodense solid necrosis may also have a central alveolus varying in size as a sign of vitality. The results also emphasise the significance of the terminology selected for the EMUC-CT, using the term "cystoid" rather than "cystic". 
Acknowledgements. The results included data and results from a German Research Foundation (DFG)-funded project called "Establishment of a national database for alveolar echinococcosis" (Ref. No. KA 4356/3-1 and Ref. No. KR 5204/1-2), and Müller Holding Ltd. \& Co. KG Ulm.

Members of the Echinococcosis Working Group Ulm: Thomas FE Barth, Sven Baumann, Ambros J Beer, Meinrad Beer, Johannes Bloehdorn, Agata Engler, Tilmann Graeter, Johannes Grimm, Beate Gruener, Doris Henne-Bruns, Andreas Hillenbrand, Katharina Klein, Wolfgang Kratzer, Patrycja Schlingeloff, Julian Schmidberger, Rong Shi, Frauke Theis.

\section{Conflict of interest}

The authors declare that they have no conflict of interest.

\section{References}

1. Ammann RW, Eckert J. 1996. Cestodes. Echinococcus. Gastroenterology Clinics of North America, 25, 655-689.

2. Aoki T, Hagiwara M, Yabuki H, Ito A. 2015. Unique MRI findings for differentiation of an early stage of hepatic alveolar echinococcosis. BMJ Case Reports, bcr2014208123.

3. Azizi A, Blagosklonov O, Lounis A, Berthet L, Vuitton DA, Bresson-Hadni S, Delabrousse E. 2015. Alveolar echinococcosis: correlation between hepatic MRI findings and FDG-PET/CT metabolic activity. Abdominal Imaging, 40, 56-63.

4. Bresson-Hadni S, Delabrousse E, Blagosklonov O, Bartholomot B, Koch S, Miguet JP, Mantion GA, Vuitton DA. 2006. Imaging aspects and non-surgical interventional treatment in human alveolar echinococcosis. Parasitology International, 55, 267-272.

5. Brunetti E, Kern P, Vuitton DA. 2010. Expert consensus for the diagnosis and treatment of cystic and alveolar echinococcosis in humans. Acta Tropica, 114, 1-16.

6. Buttenschoen K, Carli Buttenschoen D, Gruener B, Kern P, Beger HG, Henne-Bruns D, Reuter S. 2009. Long-term experience on surgical treatment of alveolar echinococcosis. Langenbeck's Archives of Surgery, 394, 689-698.

7. Carrim ZI, Murchison JT. 2003. The prevalence of simple renal and hepatic cysts detected by spiral computed tomography. Clinical Radiology, 58, 626-629.

8. Craig P, Rogan MT, Campos-Ponce M. 2003. Echinococcosis: disease, detection and transmission. Parasitology, 127, 5-20.

9. Didier D, Weiler S, Rohmer P, Lassegue A, Deschamps JP, Vuitton D, Miguet JP, Weill F. 1985. Hepatic alveolar echinococcosis: correlative US and CT study. Radiology, 154, 179-186.

10. Eckert J, Deplazes P. 2004. Biological, epidemiological, and clinical aspects of echinococcosis, a zoonosis of increasing concern. Clinical Microbiology Reviews, 17, 107-135.

11. Gaines PA, Sampson MA. 1989. The prevalence and characterization of simple hepatic cysts by ultrasound examination. British Journal of Radiology, 62, 335-337.

12. Graeter T, Kratzer W, Oeztuerk S, Haenle MM, Mason RA, Hillenbrand A, Kull T, Barth TF, Kern P, Gruener B. 2016. Proposal of a computed tomography classification for hepatic alveolar echinococcosis. World Journal of Gastroenterology, 22, 3621-3631.

13. Graeter T, Bao H, Delabrousse E, Brumpt E, Shi R, Li W, Jiang Y, Liu W, XUUB Consortium. 2019. Hepatic alveolar echinococcosis: the first intercontinental comparative study between China and Europe. (Submitted).
14. Graeter T, Shi R, Leitner E, Kratzer W, Barth TF, Hillenbrand A, Henne-Bruns D, Schmidberger J, Gruener B. 2019. Long-term changes in focal lesions in hepatic alveolar echinococcosis treated with benzimidazoles using the EMUC-CT: a retrospective cohort study. (Submitted).

15. Hounsfield GN. 1973. Computerized transverse axial scanning (tomography). 1. Description of system. British Journal of Radiology, 46, 1016-1022.

16. Kadry Z, Renner EC, Bachmann LM, Attigah N, Renner EL, Ammann RW, Clavien PA. 2005. Evaluation of treatment and long-term follow-up in patients with hepatic alveolar echinococcosis. British Journal of Surgery, 92, 1110-1116.

17. Kaltenbach TE, Engler P, Kratzer W, Oeztuerk S, Seufferlein T, Haenle MM, Graeter T. 2016. Prevalence of benign focal liver lesions: ultrasound investigation of 45,319 hospital patients. Abdominal Radiology, 41, 25-32.

18. Kantarci M, Bayraktutan U, Karabulut N, Aydinli B, Ogul H, Yuce I, Calik M, Eren S, Atamanalp SS, Oto A. 2012. Alveolar echinococcosis: spectrum of findings at cross-sectional imaging. Radiographics, 32, 2053-2070.

19. Kern P, Bardonnet K, Renner E, Auer H, Pawlowski Z, Ammann RW, Vuitton DA. 2003. European echinococcosis registry: human alveolar echinococcosis, Europe, 1982-2000. Emerging Infectious Diseases, 9, 343-349.

20. Kern P, Wen H, Sato N, Vuitton DA, Gruener B, Shao Y, Delabrousse E, Kratzer W, Bresson-Hadni S. 2006. WHO classification of alveolar echinococcosis: principles and application. Parasitology International, 55, 283-287.

21. Kodama Y, Fujita N, Shimizu T, Endo H, Nambu T, Sato N, Todo S, Miyasaka K. 2003. Alveolar echinococcosis: MR findings in the liver. Radiology, 228, 172-177.

22. Kolářová L, Matějů J, Hrdý J, Kolářová H, Hozáková L, Žampachová V, Auer H, Stejskal F. 2015. Human alveolar echinococcosis, Czech Republic, 2007-2014. Emerging Infectious Diseases, 21, 2263-2265.

23. Kratzer W, Gruener B, Kaltenbach TE, Ansari-Bitzenberger S, Kern P, Fuchs M, Mason RA, Barth TF, Haenle MM, Hillenbrand A, Oeztuerk S, Graeter T. 2015. Proposal of an ultrasonographic classification for hepatic alveolar echinococcosis: Echinococcosis multilocularis Ulm classification-ultrasound. World Journal of Gastroenterology, 21, 12392-12402.

24. Lantinga MA, Gevers TJ, Drenth JP. 2013. Evaluation of hepatic cystic lesions. World Journal of Gastroenterology, 19, 3543-3554.

25. Larssen TB, Rørvik J, Hoff SR, Horn A, Rosendahl K. 2005. The occurrence of asymptomatic and symptomatic simple hepatic cysts. A prospective, hospital-based study. Clinical Radiology, 60, 1026-1029.

26. Liu YH, Wang XG, Chen YT, Yao YQ. 1993. Computer tomography of liver in alveolar echinococcosis treated with albendazole. Transactions of the Royal Society of Tropical Medicine and Hygiene, 87, 319-321.

27. Moro P, Schantz PM. 2009. Echinococcosis: a review. International Journal of Infectious Diseases, 13, 125-133.

28. Pawlowski ZS, Eckert J, Vuitton DA, Ammann RW, Kern P, Craig PS, et al. 2001. Echinococcosis in humans: clinical aspects, diagnosis and treatment, in WHO/OIE manual on echinococcosis in humans and animals, Eckert J, Gemmel MA, Meslin F-X, Pawlowski Z, Editors. Paris' World Organization for Animal Health and World Health Organization: Paris. p. 20-71.

29. Poetter-Lang S, Brancatelli G, Bastati-Huber N, Ba-Ssalamah A. 2015. Moderne Diagnostik zystischer Leberläsionen und Hämangiome. Radiologe, 55, 9-17.

30. Qian LJ, Zhu J, Zhuang ZG, Xia Q, Liu Q, Xu JR. 2013. Spectrum of multilocular cystic hepatic lesions: CT and MR 
imaging findings with pathologic correlation. Radiographics, 33, 1419-1433.

31. Reuter S, Nüssle K, Kolokythas O, Haug U, Rieber A, Kern P, Kratzer W. 2001. Alveolar liver echinococcosis: a comparative study of three imaging techniques. Infection, 29, $119-125$.

32. Reuter S, Buck A, Manfras B, Kratzer W, Seitz HM, Darge K, Reske SN, Kern P. 2004. Structured treatment interruption in patients with alveolar echinococcosis. Hepatology, 39, 509-517.

33. Sade R, Kantarci M, Genc B, Ogul H, Gundogdu B, Yilmaz O. 2018. Computed tomography perfusion imaging for the diagnosis of hepatic alveolar echinococcosis. Eurasian Journal of Medicine, 50, 1-5.

34. Sade R, Kantarci M, Ogul H, Gundogdu B, Aydinli B. 2018. Differentiation between hepatic alveolar echinococcosis and primary hepatic malignancy with diffusion-weighted magnetic resonance imaging. Diagnostic and Interventional Imaging, 99, 169-177.
35. Sanfelippo PM, Beahrs OH, Weiland LH. 1974. Cystic disease of the liver. Annals of Surgery, 179, 922-925.

36. Voelk M, Strotzer M, Lenhart M, Techert J, Seitz J, Feuerbach S. 2001. Frequency of benign hepatic lesions incidentally detected with contrast-enhanced thin-section portal venous phase spiral CT. Acta Radiologica, 42, 172-175.

37. Wang Q, Qiu J, Yang W, Schantz PM, Raoul F, Craig PS, Giraudoux P, Vuitton DA. 2006. Socioeconomic and behavior risk factors of human alveolar echinococcosis in Tibetan communities in Sichuan, People's Republic of China. American Journal of Tropical Medicine and Hygiene, 74, 856-862.

38. Wuestenberg J, Gruener B, Oeztuerk S, Mason RA, Haenle MM, Graeter T, Akinli AS, Kern P, Kratzer W. 2014. Diagnostics in cystic echinococcosis: serology versus ultrasonography. Turkish Journal of Gastroenterology, 25, 398-404.

39. Yamasaki H, Nakaya K, Nakao M, Sako Y, Ito A. 2007. Significance of molecular diagnosis using histopathological specimens in cestode zoonoses. Tropical Medicine and Health, $35,307-321$.

Cite this article as: Engler A, Shi R, Beer M, Schmidberger J, Kratzer W, Barth TF, Grimm J, Hillenbrand A, Henne-Bruns D, Gruener B, Beer AJ \& Graeter T. 2019. Simple liver cysts and cystoid lesions in hepatic alveolar echinococcosis: a retrospective cohort study with Hounsfield analysis. Parasite 26, 54.

\section{PARASTE}

An international open-access, peer-reviewed, online journal publishing high quality papers on all aspects of human and animal parasitology

Reviews, articles and short notes may be submitted. Fields include, but are not limited to: general, medical and veterinary parasitology; morphology, including ultrastructure; parasite systematics, including entomology, acarology, helminthology and protistology, and molecular analyses; molecular biology and biochemistry; immunology of parasitic diseases; host-parasite relationships; ecology and life history of parasites; epidemiology; therapeutics; new diagnostic tools.

All papers in Parasite are published in English. Manuscripts should have a broad interest and must not have been published or submitted elsewhere. No limit is imposed on the length of manuscripts.

Parasite (open-access) continues Parasite (print and online editions, 1994-2012) and Annales de Parasitologie Humaine et Comparée (1923-1993) and is the official journal of the Société Française de Parasitologie. 